\title{
Holomorphic Foliations and Kupka Singular Sets
}

\author{
B. Azevedo Scárdua \& C. Camacho
}

Let $\mathcal{F}$ be a nondicritical codimension one holomorphic foliation on the $k$-dimensional complex projective space $M=\mathbb{C} P(k), k \geq 3$. Assume that the closure $\overline{K(\mathcal{F})}$ of the Kupka singular set is a complete intersection projective variety and that the points in $\overline{K(\mathcal{F})}-K(\mathcal{F})$ exhibit slightly generic holomorphic integrating factors. Then the Kupka components have normalizable transverse type and therefore the foliation $\mathcal{F}$ is given by a closed rational 1 form on $\mathbb{C} P(k)$. In general (for $M$ a complex manifold and $K(\mathcal{F})$ not necessarily complete intersection), we prove the existence of an affine or projective transverse structure in a neighborhood of the Kupka singular set.

\section{Introduction.}

\subsection{Singular holomorphic foliations.}

According to Frobenius Theorem [23] a (nonsingular) codimension one holomorphic foliation $\mathcal{F}$ on a complex manifold $M$ is given by an open covering $M=\bigcup_{j \in J} U_{j}$ of $M$ such that in each $U_{j}$ we have a nonsingular holomorphic 1 -form $\omega_{j}$ that satisfies the integrability condition $\omega_{j} \wedge d \omega_{j}=0$, and such that for each $U_{j} \cap U_{i} \neq \emptyset$ we have $\left.\omega_{i}\right|_{U_{i} \cap U_{j}}=\left.g_{i j} \cdot \omega_{j}\right|_{U_{i} \cap U_{j}}$ for some holomorphic nonvanishing function $g_{i j}$ in $U_{i} \cap U_{j}$. If $M$ is a complex projective space $M=\mathbb{C} P(k)$ then it is known that there are no such foliations without singularities. On the other hand we may consider singular holomorphic foliations defined as follows: A singular codimension one holomorphic foliation on $M$ is defined by an open cover $M=\bigcup_{j \in J} U_{j}$ and integrable holomorphic 1-forms as above, but that may have singularities. The singular

\footnotetext{
${ }^{1}$ Scárdua was supported by CNPq-Brasil and Min.Affaires Étrangerès-France.
} 
set $\operatorname{sing} \omega_{j}=\left\{p \in U_{j}, \omega_{j}(p)=0\right\}$ is an analytic subset of $U_{j}$ that satisfies: sing $\omega_{j} \cap U_{i}=\operatorname{sing} \omega_{i} \cap U_{j}$ for all $U_{i} \cap U_{j} \neq \emptyset$. This shows that there exists an analytic subset $S \subset M$ such that $S \cap U_{j}=\operatorname{sing} \omega_{j}$ and by the first case the open manifold $M^{\prime}=M \backslash S$ is foliated by a codimension one (nonsingular) holomorphic foliation $\mathcal{F}^{\prime}$. Using Hartogs' Extension Theorem [9],[10] it is possible to prove that $\mathcal{F}^{\prime}$ extends in an unique way as a foliation on $M \backslash S_{2}$ where $S_{2} \subset S$ is a codimension two analytic subset of $M$ that is chosen to be minimal with this extension property. In other words, we may assume that the singular set $S$ has codimension two in $M$. In this case we define $\operatorname{sing} \mathcal{F}=S$ and $\mathcal{F}$ will be the pair $\left(\mathcal{F}^{\prime}\right.$, sing $\left.\mathcal{F}\right)$. We call $\mathcal{F}$ a codimension one singular holomorphic foliation on $M$ and $\operatorname{sing} \mathcal{F}$ is the singular set of $\mathcal{F}$.

\subsection{Foliations on projective spaces.}

We turn our attention to the case $M$ is a projective space $\mathbb{C} P(k)$ of dimension $k \geq 2$. Using the triviality of certain cohomology groups on $\mathbb{C}^{k+1} \backslash\{0\}$ (see Cartan's Theorem and the solution of the Cousin Problems in $\mathbb{C}^{k+1} \backslash\{0\}$ in [8] [10]) it can be shown that a codimension one holomorphic foliation on a complex projective space $\mathbb{C} P(k)$ can be defined in homogeneous coordinates in $\mathbb{C}^{k+1}$ by a differential 1 -form

$$
\omega=\sum_{i=1}^{k+1} a_{i}(z) d z_{i} \quad z \in \mathbb{C}^{k+1}
$$

where the $a_{i}(z)$ are homogeneous polynomials of the same degree (without common factors and) satisfying $\sum_{i=1}^{k+1} z_{i} a_{i}(z)=0$, and the integrability condition $\omega \wedge d \omega=0$. The singular set of $\omega$ is $S(\omega):=\left\{z \in \mathbb{C}^{k+1} ; a_{j}(z)=\right.$ $0 j=1, \ldots, k+1\}$. We denote by $\widehat{\mathcal{F}}(\omega)$ the codimension one singular holomorphic foliation on $\mathbb{C}^{k+1}$ defined by $\omega$ with singular set $\operatorname{sing} \omega$. If $\pi: \mathbb{C}^{k+1} \backslash\{0\} \rightarrow \mathbb{C} P(k)$ denotes the canonical projection, this induces a foliation $\mathcal{F}=\mathcal{F}(\omega)$ on $\mathbb{C} P(k)$ with singular set sing $\mathcal{F}=\pi(S(\omega))$ and $\pi^{*}(\mathcal{F})=\widehat{\mathcal{F}}(\omega)$. As we have remarked above there is no loss of generality if we assume that cod sing $\mathcal{F} \geq 2$. Let us first comment the case $k=2$.

\subsection{Singularities in dimension 2 .}

In the two dimensional case the singular set is discrete and we regard the local behaviour of $\mathcal{F}$ around an isolated singular point. We may therefore consider a germ of holomorphic 1-form $\omega(x, y)=A(x, y) d x+B(x, y) d y$ 
with an isolated singularity at the origin $0 \in \mathbb{C}^{2}$ or the dual vector field $X=(-B, A)$. Let us recall some basic facts [2],[16]:

- A separatrix is a germ of irreducible analytic curve which is invariant and passes through the singularity.

- The singularity is called nondenegerate if the linear part $D X(0)$ is nonsingular. In this case we may write $\mathcal{F}: x d y-\lambda y d x+$ h.o.t. $=0$ (where h.o.t. means "higher order terms"), for some $\lambda \in \mathbb{C}^{*}$. The class of nondegenerate singularities can be "stratified" as follows.

The singularity is hyperbolic if $\lambda \notin \mathbb{R}$. Hyperbolicity implies linearization of the foliation around the singular point [16].

If $\lambda \in \mathbb{C} \backslash \mathbb{R}_{\text {— }}$ the singularity is in the Poincaré domain. This is equivalent to the following geometrical condition: the leaves of $\mathcal{F}$ are transverse to the small 3-spheres $\mathbb{S}_{\epsilon}^{3}(0)$ centered at the singularity. A Poincare singularity is resonant if $\left\{\lambda, \lambda^{-1}\right\} \cap \mathbb{N} \neq \emptyset$. A nonresonant Poincaré type singularity is analytically linearizable [16]. In the resonant case the singularity is either analytically linearizable or can be put in the Poincaré-Dulac form: $x d y-$ $\left(n y+x^{n}\right) d x=0$ [14]. In this last case there exists only one separatrix, its holonomy map is tangent to the identity and nonperiodic.

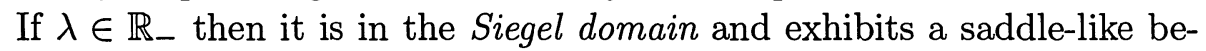
haviour: there are exactly two (transverse and smooth) separatrices and if a local open leaf (that is, a leaf which is not a separatrix) accumulates the singularity then it accumulates both separatrices. When $\lambda \in \mathbb{Q}_{-}$the singularity will be called resonant. An important subclass of Siegel resonant singularities is the class of the ones exhibiting holomorphic first integrals. This class is characterized by the topological condition that the leaves are closed outside the singularity [16]. In general a resonant Siegel singularity is not linearizable, and there is no standart model to which it is analytically conjugate. On the other hand there are formal (nonlinear) models given by Martinet-Ramis [14], that is, models to which any nonlinearizable singularity is formally conjugate. If the Siegel singularity is nonresonant then it is formally linearizable [16].

- In general we have the Reduction Theorem of Seidenberg [2],[16] that asserts the existence of a proper holomorphic map $\pi: \widetilde{U} \rightarrow U$ (for a fixed small enough neighborhood $U$ of the origin) which is a finite composition of quadratic blow-up's at singular points such that the foliation $\pi^{*} \mathcal{F}$ pull-back of $\mathcal{F}$ by $\pi$, exhibits in $\pi^{-1}(0)$ only irreducible singularities which can be of the following two types:

(i) $x d y-\lambda y d x+$ h.o.t. $=0$ and $\lambda \notin \mathbb{Q}_{+}$(simple singularities),

(ii) $y^{p+1} d x-\left[x\left(1+\lambda y^{p}\right)+\right.$ h.o.t. $] d y=0, p \geq 1$. This last case is called saddle-node. We call $(y=0)$ the strong separatrix of the saddle-node. 
The divisor $\mathrm{D}=\pi^{-1}(0) \subset \widetilde{M}$ of the resolution $\pi$ can be written as $D=$ $\bigcup_{j=1}^{m} D_{j}$, where each $\left\{D_{j}\right\}$ is diffeomorphic to the projective line $\mathbb{C} P(1)$.

Definition 1. The singularity $0 \in U$ is nondicritical if $D=\pi^{-1}(0)$ is invariant by $\mathcal{F}^{*}$ and this is equivalent to the fact that $\mathcal{F}$ exhibits only a finite number of separatrices passing through the fixed singularity. If a nondegenerate singularity is dicritical then it is of the form $n x d y-m y d x+$ h.o.t. $=$ $0, n, m \in \mathbb{N}$.

\subsection{Kupka singularities.}

Now we regard the case $k \geq 3$. In this case the singularities may be no longer isolated. However we may expect to have some local product structure (of a regular foliation by a two dimensional singularity). This is done by means of the study of the Kupka phenomena that we proceed to describe. In the singular set of $\mathcal{F}$ we can distinguish the Kupka singular subset defined as $K(\mathcal{F})=\pi(K(\omega))$ where $K(\omega)=\left\{p \in \mathbb{C}^{k+1} \backslash\{0\} ; \omega(p)=0\right.$ and $d \omega(p) \neq$ $0\}$ [11]. The main properties of the Kupka subset are summarized in the following theorem (see [5],[7],[11],[13],[17],[18]).

Theorem 1.1. Let $k \geq 3, \mathcal{F}, \omega, K(\mathcal{F})$ be as above:

(i) The Kupka set $K(\mathcal{F})$ is a locally closed codimension 2 smooth submanifold of $\mathbb{C} P(k)$.

(ii) The Kupka set has the local product structure: Given a connected component $K \subset K(\mathcal{F})$ there exist a holomorphic 1-form $\Theta$, called the transversal type of $K$, defined on a neighborhood of $0 \in \mathbb{C}^{2}$ and vanishing only at 0 , a covering $\left\{U_{\alpha}\right\}$ of a neighborhood of $K$ in $\mathbb{C} P(k)$ and a family of holomorphic submersions $\varphi_{\alpha}: U_{\alpha} \rightarrow \mathbb{C}^{2}$ satisfying: $\varphi_{\alpha}^{-1}(0)=K \cap U_{\alpha}, \varphi_{\alpha}^{*} \Theta$ defines $\mathcal{F}$ in $U_{\alpha}$.

(iii) $K(\mathcal{F})$ is persistent under small perturbations of $\mathcal{F}$, namely, fixed any $p \in K(\mathcal{F})$ with defining 1-form $\varphi^{*} \Theta$ as above, and for any foliation $\mathcal{F}^{\prime}$ sufficiently close to $\mathcal{F}$, there is a holomorphic 1-form $\Theta^{\prime}$ close to $\Theta$ and a submersion $\varphi^{\prime}$ close to $\varphi$, such that $\mathcal{F}^{\prime}$ is defined by $\left(\varphi^{\prime}\right)^{*} \Theta^{\prime}$ near the point $p$.

(iv) Let $K \subset K(\mathcal{F})$ be a compact connected component such that the first Chern class of the normal bundle of $K$ in $\mathbb{C} P(k)$ is nonzero, then the transversal type of $K$ is $\Theta(x, y)=n x d y-m y d x, n, m \in \mathbb{N}$ and this transversal type is constant through analytic deformations of the foliation. 
On the other hand, not much is known about the structure of $\mathcal{F}$ near a singular point not lying in the Kupka set. ${ }^{2}$ An important result along this direction is the Reeb theorem [19] that states that if $w(q)=0, d w(q)=0$ and the linear part of $w$ at $q$ is nondegenerated then, near $q$, the foliation $\mathcal{F}$ has a first integral which is a Morse function. In dimension three it is known also that if $q \in \operatorname{sing} \mathcal{F}$ is an isolated common zero of $w$ and $d w$ then near $q$ the leaves of $\mathcal{F}$ are the orbits of a 2- dimensional Lie group action $[1],[12]$.

Definition 2. Let $K \subset K(\mathcal{F})$ be an irreducible component of $K(\mathcal{F})$ and $\Theta=A(x, y) d x+B(x, y) d y=0$ its transversal type.

- We say that $K$ is in the Poincaré domain if the first jet of $\Theta$ is of the form $\lambda x d y-\mu y d x, \lambda / \mu \in \mathbb{C} \backslash \mathbb{R}_{-}$. In this case we say that it is resonant if either $\lambda / \mu \in \mathbb{N}$ or $\mu / \lambda \in \mathbb{N}$. We say that $K$ is in the Siegel domain if $\lambda / \mu \in \mathbb{R}_{-}$. In this last case we say it is resonant if $\lambda / \mu \in \mathbb{Q}_{-}$(see section $\mathbf{1 . 3}$ above).

- $K$ is analytically normalizable if $\Theta$ is either: (i) in the Poincaré Domain, $\Theta=x d y-\lambda y d x+$ h.o.t., $\lambda \in \mathbb{C} \backslash \mathbb{R}_{-}$. (ii) resonant in the Siegel domain being linearizable or , $\Theta=x d y-\lambda y d x, \lambda \in \mathbb{R}_{-}$. (iii) in the Siegel domain resonant but analytically normalizable, i.e., analytically conjugate to the formal normal form of Martinet-Ramis ([14]). (iv) a saddle-node analytically conjugated to the normal form $\Theta=x\left(1+\lambda y^{k}\right) d y-y^{k+1} d x$ [15].

- A singularity $q \in \overline{K(\mathcal{F})} \backslash K(\mathcal{F})$ is called nonresonant if there exists at least one component $K \subset K(\mathcal{F}), q \in \bar{K}$, of type (i) or (ii) that is nonresonant.

\subsection{Statement of the main results.}

First we recall the definition of complex complete intersection we use [5],[24]: Let $S \subset M^{k}$ be an analytic subvariety of codimension $q$.

Definition 3. We say that $S$ is a complete intersection if there exist codimension one analytic submanifolds $S_{1}, \ldots, S_{q}$; such that $S=S_{1} \cap \cdots \cap S_{q}$ with transverse intersection off some codimension $\geq 1$ subset of $S . \quad 3 \quad$ A complete intersection will be called a transverse complete intersection if the intersection $S_{1} \cap \cdots \cap S_{q}$ is everywhere transverse.

\footnotetext{
${ }^{2}$ For instance it is an open question to classify the foliations with empty Kupka set.

${ }^{3}$ In particular any algebraic curve in $\mathbb{C} P(2)$ (possibly singular) is a complete intersection.
} 
The main property we use concerning complete intersections is the following theorem of E.Levi (see [5], [24]):

Theorem 1.2. Let $S \subset \mathbb{C P}(k)$ be a codimension $q$ complete intersection algebraic submanifold. Given a neighborhood $V$ of $S$ in $\mathbb{C} P(k)$ any meromorphic 1-form $w$ defined on $V$ extends meromorphically (i.e., rationally) to $\mathbb{C} P(k)$. In fact, the complementary $M=\mathbb{C} P(k) \backslash S$ is a $(k-q+1)$-complete complex manifold in the sense of [5],[24].

Next we give some remarkable examples of foliations and Kupka sets.

Example 1 (Darboux foliations). A Darboux 1-form (see [4], [20] where Darboux 1-forms are called logarithmic 1-forms) is given in $\mathbb{C}^{k+1}$ by

$$
\mathcal{D}=\sum_{j=1}^{k+1} \lambda_{j} f_{1} \ldots \hat{f}_{j} \ldots f_{k+1} d f_{j}, \quad \lambda_{j} \in \mathbb{C}
$$

where the $f_{j}$ 's are homogeneous polynomials in $k+1$ complex variables. Clearly $\mathcal{D}$ admits the integrating factor $1 / f$ where $f=f_{1} \ldots f_{k+1}$, i.e., the linear 1 -form $\frac{1}{f} \mathcal{D}$ is closed. The 1 -form $\mathcal{D}$ can be seen also as the pull back by the map $F=\left(f_{1}, \ldots, f_{k+1}\right)$ of the 1 -form

$$
\mathcal{L}=\sum_{j=1}^{k+1} \lambda_{j} x_{1} \ldots \hat{x}_{j} \ldots x_{k+1} d x_{j}
$$

where $\left(x_{1}, \ldots, x_{k+1}\right)$ are affine coordinates for $\mathbb{C}^{k+1}$.

Now we take a polynomial Darboux 1 -form $\mathcal{D}$ in $\mathbb{C}^{k+1}$ as above. The set $\operatorname{sing}(\mathcal{L})$ is the union of all codimension two coordinate planes and $\overline{K(\mathcal{L})} \backslash K(\mathcal{L})$ is the union of all codimension three coordinate planes. A straighforward calculation shows that the Kupka set $K(\mathcal{D}) \subset F^{-1}(K(\mathcal{L}))$ and moreover there exists a codimension 2 analytic subset $S \subset \operatorname{sing} \mathcal{F}$ satisfying:

(a) $S$ is a complete intersection variety i.e. $S=\left\{[z] \in \mathbb{C} P(k) ; h_{1}(z)=\right.$ $\left.h_{2}(z)=0\right\}$ where $h_{1}$ and $h_{2}$ are homogeneous polynomials in $\mathbb{C}^{k+1}$.

(b) $K(\mathcal{F}) \cap S$ is open and dense in $S$.

(c) Given $q \in S \backslash K(\mathcal{F}), \quad \mathcal{F}$ can be represented in a neighborhood of $q$ by a closed meromorphic 1-form $\Omega_{q}$.

If for instance $\lambda_{j} / \lambda_{i} \notin \mathbb{Q}, \forall i \neq j$ then we also have the following condition:

(d) Given $K \subset K(\mathcal{F})$ the transverse type $\Theta$ admits no meromorphic first integral. 
Example 2 (Closed meromorphic 1-forms). The general form of a closed rational 1-form $\eta$ in $\mathbb{C}^{k}$, with polar divisor the zero set of a polynomial function $f$ is,

$$
\eta=\sum_{j=1}^{r} \lambda_{j} \frac{d f_{j}}{f_{j}}+d\left(\frac{g}{\prod_{j=1}^{r} f_{j}^{n_{j}-1}}\right)
$$

where $f=f_{1}^{n_{1}} \ldots f_{r}^{n_{r}}$ is its decomposition in irreducible factors and $\lambda_{j} \in \mathbb{C}$ (see [4]). This result is called Integration Lemma. An analogous expression exists in the local analytic case (see [6]). Using these local expressions one can verify that again the Kupka set may verify conditions (a), (b), (c) and (d) above if we have for instance $\lambda_{i} / \lambda_{j} \notin \mathbb{Q}, \forall i \neq j$.

Now we recall $[\mathbf{2 0}],[\mathbf{2 1}]$ :

Definition 4. Given a meromorphic integrable 1-form $\omega$ on $M$ we say that $(\omega, \eta)$ is an affine pair if:

(1) $d \omega=\eta \wedge \omega, d \eta=0$

(2) the polar divisor $(\eta)_{\infty}$ of $\eta$ has order one and consists of the union of $(\omega)_{\infty} \cup(\omega)_{0}$ and an invariant divisor of $\mathcal{F}$,

(3) the residue of $\eta$ along any noninvariant irreducible component $C$ of $(\omega)_{\infty} \cup(\omega)_{0}$ is equal to either - (the order of the poles of $\omega$ along $C$ ), or (the order of $(\omega)_{0}$ along $\left.C\right)$, respectively.

Definition 5. Let $\mathcal{F}$ be a foliation given by an integrable meromorphic 1form $\omega$ on $M$ (not necessarily with isolated singularities). We assume that $(\omega)_{0} \cup(\omega)_{\infty}$ has no $\mathcal{F}$-invariant component and that there exists a meromorphic 1-form $\eta$ (usually non closed) such that $d \omega=\eta \wedge \omega$ (see Remark 2 (iv)). A meromorphic 1 -form $\xi$ is such that $(\omega, \eta, \xi)$ defines a projective triple for $\mathcal{F}$, if: (1) $\omega=\eta \wedge \omega$, with $(\eta)_{\infty}=(\omega)_{\infty} \cup(\omega)_{0},(\eta)_{\infty}$ of order one and such that for any irreducible component $C \subset(\omega)_{\infty} \cup(\omega)_{0}$ we have either $\operatorname{Res}_{C} \eta=-\operatorname{ord}\left((\omega)_{\infty}, C\right)$, or $\operatorname{Res}_{C} \eta=\operatorname{ord}\left((\omega)_{0}, C\right)$, respectively. (2) $d \eta=\omega \wedge \xi$, (3) $d \xi=\xi \wedge \eta,(\xi)_{0}=(\omega)_{\infty}$ and $(\xi)_{\infty}=(\omega)_{0}$ and for each irreducible component $C \subset(\omega)_{\infty} \cup(\omega)_{0}$ we have either $\operatorname{ord}\left((\xi)_{0}, C\right)=\operatorname{ord}\left((\omega)_{\infty}, C\right), \operatorname{or} \operatorname{ord}\left((\xi)_{\infty}, C\right)=\operatorname{ord}\left((\omega)_{0}, C\right)$ respectively.

Remark 1. The existence of an affine pair implies the existence of a Liouvillian first integral $F=\int\left(\omega / \exp \left(\int \eta\right)\right)$, for the foliation $\mathcal{F}$ defined by $\omega$, and is also equivalent to the existence of an affine transverse structure outside some algebraic nivariant codimension one subset (see [20],[21]). The 
existence of a projective triple is equivalent to the existence of a projective transverse structure for $\mathcal{F}$ (the foliation defined by $\omega$ ), on a certain open subset $U=\mathbb{C} P(k) \backslash\left((\eta)_{\infty} \cup(\xi)_{\infty}\right)$ (see [20], [21] for a detailed description).

Our main results are the following:

Theorem A. Let $\mathcal{F}$ be a holomorphic foliation on $\mathbb{C} P(k), k \geq 3$, such that there exists a codimension 2 algebraic subset $S \subset \operatorname{sing} \mathcal{F}$ satisfying conditions (a),(b),(c) and (d). Then $\mathcal{F}$ is given by a closed rational 1form on $\mathbb{C} P(k)$.

Theorem B. Let $\mathcal{F}$ be a holomorphic foliation on a $k$-dimensional complex manifold $M^{k}, k \geq 3$, such that there exists a codimension 2 analytic subset $S \subset \operatorname{sing} \mathcal{F}$ satisfying conditions (b),(c) and (d). Assume that $\mathcal{F}$ is given by a meromorphic integrable 1-form $\omega$ outside the polar set $(\omega)_{\infty}$. Then $\mathcal{F}$ admits an affine pair $(\omega, \eta)$ in a neighborhood of $S$. If $M-S$ is 2-complete then $\eta$ extends meromorphically to $M$.

Theorem C. Let $\mathcal{F}$ be a holomorphic foliation on a $k$-dimensional complex manifold $M^{k}, k \geq 3$ and given by a meromorphic integrable 1 -form $\omega$ admitting a 1-form $\eta$ such that $d \omega=\eta \wedge \omega$. Assume that there exists an irreducible codimension 2 analytic subset $S \subset \operatorname{sing} \mathcal{F}$ satisfying condition (b) and the following condition: (e) $S \cap K(\mathcal{F})$ is a certain Kupka component $K$ which has a transverse type admitting a meromorphic first integral.

Then $\mathcal{F}$ admits a projective triple $(\omega, \eta, \xi)$; defined in a neighborhood of $S$.

Remark 2. A few words should be said related to the hypothesis we make in Theorems A, B and C above.

(i) Theorem A generalizes one of the main results of [5].

(ii) According to [3], the existence of the closed form $\Omega_{q}$ required in condition (c) is always fulfilled if the germ of singularity $q \in \operatorname{sing}(\mathcal{F}) \backslash K(\mathcal{F})$ can be desingularized with ordinary (punctual) blow-ups. In [3] one can also find a connectivity hypothesis on the germ of $\operatorname{sing}(\mathcal{F})$ at $q$, which implies the existence of a closed meromorphic $\Omega_{q}$ as in condition (d).

(iii) The hypothesis of complete intersection for $S$ is essential and cannot be removed. A counterexample can be obtained as follows: Take a foliation $\mathcal{F}_{o}$ with hyperbolic singularities in $\mathbb{C} P(2)$. Let $P: \mathbb{C} P(3) \rightarrow \mathbb{C} P(2)$ be a linear submersion in general position with respect to $\mathcal{F}_{o}$. Then the pullback foliation $\mathcal{F}:=P^{*}(\mathcal{F})$ on $\mathbb{C} P(3)$ exhibits singularities that are either of kupka type or isolated non kupka singularities that are also simple in the 
sense of [1]. In particular we may choose $P$ and $\mathcal{F}_{o}$ in such a way that conditions (b),(c) and (d) are fullfiled for a suitable $S \subset \operatorname{sing} \mathcal{F}$. However, since $\mathcal{F}_{o}$ is generic it is not given by a closed rational 1 -form and $\mathcal{F}$ may not satisfy the conclusion of Theorem A.

(iv) Given a codimension one foliation $\mathcal{F}$ on a projective (algebraic) manifold $M^{k}$ there exists an integrable rational 1-form $\omega$ on $M$ such that $\mathcal{F}$ is defined by $\omega$ in $M-(\omega)_{\infty}$. Now, given such a 1 -form $\omega$ there exists a 1 -form $\eta$ such that $d \omega=\eta \wedge \omega[20]$. In fact the existence is trivial if $k=2$ and is a consequence of the integrability of $\omega$ for $k \geq 3$.

Acknowledgments. This work was partially conceived during a postdoctorade of the first author at the Universite de Rennes I - IRMAR. He expresses his gratitude to the IRMAR and specially to D. Cerveau for the kind hospitality. The authors are grateful to the referee for his reading and suggestions. We thank O. Calvo for valuable conversations.

\section{The Kupka Set.}

Let $\mathcal{F}$ be a codimension one singular holomorphic foliation on a $k$ dimensional complex manifold $M$, given by an integrable meromorphic 1 form $\omega$ outside the polar set of $\omega$.

Lemma 2.1. Let $K \subset K(\mathcal{F})$ be a nondicritical Kupka component of $\mathcal{F}$. Assume that for a point $q \in \bar{K}-K$ there exists a meromorphic closed 1 form $\Omega_{q}$ which defines $\mathcal{F}$ in a neighborhood of $q$, minus its polar set $\left(\Omega_{q}\right)_{\infty}$. Then $K$ is analytically normalizable.

Proof. Denote by $\Theta=A(x, y) d x+B(x, y) d y$ the transverse type of $\mathcal{F}$ at $K$. Then $\Theta=0$ defines a germ of reduced singular foliation $\left(\mathcal{F}_{K}\right)^{\perp}$ at the origin $0 \in \mathbb{C}^{2}$. According to [2] there exists a germ separatrix $W$ of $\left(\mathcal{F}_{K}\right)^{\perp}$, through this singularity $0 \in \mathbb{C}^{2}$. We can assume that $W=(y=0)$, because $\Theta=0$ is reduced. Moreover we will also take $W$ to be the strong manifold if $\left(\mathcal{F}_{K}\right)^{\perp}$ is a saddle-node. First we assume that $\Theta$ is nondegenerate and in the Poincaré domain. In this case by Poincaré-Dulac Theorem it follows that $\left(\mathcal{F}_{K}\right)^{\perp}$ is either analytically linearizable and diagonalizable or it can be put in the Poincaré-Dulac form $\Theta=y d x-\left(n x+y^{n}\right) d y, n \in \mathbb{N}$. In both cases it is analytically normalizable by Definition 2. Assume now that $\Theta$ is either nondegenerate in the Siegel domain or it is a saddle-node. According to [14], [15] and [16] the holonomy of this separatrix classifies analytically this germ of foliation. But since the germ of foliation of $\mathcal{F}$ at $q$ is defined by a closed meromorphic 1-form it follows that the form $\Theta$ admits a 
holomorphic integrating factor say, $h(x, y) \in \mathcal{O}_{2}\{x, y\}$ such that $d\left(\frac{1}{h} \Theta\right)=0$. This implies that the holonomy local diffeomorphism $\varphi(y)$ associated to $W$ is either analytically linearizable or analytically normalizable in the sense of [21] and [14],[15]. Thus this implies that the germ of foliation $\left(\mathcal{F}_{K}\right)^{\perp}$ is analytically normalizable in the sense above. This proves the lemma.

When the transversal type of a component $K \subset K(\mathcal{F})$ is analytically normalizable, there exists a transverse (affine or projective) structure for $\mathcal{F}$ in a neighborhood of $K$ in $M$ minus eventually the local separatrices of $\mathcal{F}$, (see also $[20],[21])$.

Proposition 2.2. Let $k \geq 3, \mathcal{F}, K(\mathcal{F})$ be as above. Let $K \subset K(\mathcal{F})$ be a connected component with analytically normalizable transversal type $\Theta$. Then we have the following possibilities:

(1) $\Theta$ is linearizable dicritical $\Theta=n x d y-m y d x, n / m \in \mathbb{Q}_{+}$then $\mathcal{F}$ is transversely projective in a neighborhood $\mathcal{U}(K)$ of $K$ in $\mathbb{C} P(k)$.

(2) $\Theta$ is not as in (1) and nondegenerate: there exists a neighborhood $\mathcal{U}(K)$ of $K$ such that $\mathcal{F}$ is given in $\mathcal{U}(K)$ by a closed meromorphic 1-form $\Omega_{K}$, with $\left(\Omega_{K}\right)_{\infty}$ consisting of the local separatrices $\operatorname{sep}(\mathcal{F}, K)$ of $\mathcal{F}$ through $K$. (3) $\Theta$ is a normal form saddle-node $\Theta=x\left(1+\lambda y^{k}\right) d y-y^{k+1} d x$ and $\mathcal{F}$ is given by a closed meromorphic 1-form $\Omega_{K}$ in some neighborhood $\mathcal{U}(K)$ of $K$ in $\mathbb{C} P(k)$, minus the polar set which consists of the set of local separatrices $\operatorname{sep}(\mathcal{F}, K)$.

Proof. Let us assume that $k=3$, (this only simplifies the notation).

Proof of (1): It is enough to prove the following claim.

Claim 2.3. Let $(f, g),(\tilde{f}, \tilde{g}): U \rightarrow \mathbb{C}^{2}$ be holomorphic submersions such that $n f d g-m g d f=0$ and $n \tilde{f} d \tilde{g}-m \tilde{g} d \tilde{f}=0$ define the same foliation $\mathcal{F}$ on $U$. Then we have $\tilde{f}^{m} / \tilde{g}^{n}=S\left(f^{m} / g^{n}\right)$ for some Möbius transformation $S(z)=\frac{a z+b}{c z+d}$.

Proof of the Claim. The foliation $\mathcal{F}$ has $g^{n} / f^{m}$ and $\tilde{g}^{n} / \tilde{f}^{m}$ as meromorphic first integrals and has leaves of the form $\lambda \cdot g^{n}-\mu \cdot f^{m}=0$ and $\lambda \cdot \tilde{g}^{n}-\mu \cdot \tilde{f}^{m}=0$, $\lambda, \mu \in \mathbb{C}$. In particular $\{g=0\},\{\tilde{g}=0\},\{f=0\}$ and $\{\tilde{f}=0\}$ are leaves of $\mathcal{F}$. Therefore it is easy to see that there exists a Möbius transformation $S(z)=\frac{a z+b}{c z+d}, a, d, b, c \in \mathbb{C}, \quad a d-b c=1$, such that $\frac{\hat{g}^{n}}{\hat{f}^{m}}=S\left(\frac{\tilde{g}^{n}}{\tilde{f} \tilde{f}^{m}}\right)=\frac{a \tilde{g}^{n}+b \tilde{f}^{m}}{c \tilde{g}^{n}+d \tilde{f}^{m}}$ defines a meromorphic first integral for $\mathcal{F}$ and the leaves $\{\widehat{f}=0\}$ and $\{f=$ $0\}$ coincide, the same holding for the leaves $\{\widehat{g}=0\}$ and $\{g=0\}$. Now we 
only have to prove that $\widehat{g}^{n} / \widehat{f}^{m}=\lambda \cdot g^{n} / f^{m}$ for some locally constant $\lambda \in \mathbb{C}^{*}$. In fact we have $\widehat{f}=u \cdot f$ and $\widehat{g}=v \cdot g$ for some holomorphic nonvanishing $u, v$ in $U$. This implies that $\frac{v^{n}}{u^{m}}=\frac{\left(\hat{g}^{n} / \hat{f}^{m}\right)}{\left(g^{n} / f^{m}\right)}$ is a quotient of first integrals and therefore $v^{n} / u^{m}$ is a holomorphic first integral for $\mathcal{F}$ in $U$. Since $v^{n} / u^{m}$ is holomorphic we conclude that $v^{n} / u^{m}$ is locally constant in $U$. This proves the assertion and thus case (1).

Proof of (2): $\Theta=\lambda x d y-\mu y d x+$ h.o.t..

- $\Theta$ is in the Poincare domain: First we assume that $\lambda / \mu \notin \mathbb{Q}_{+}$. Then $\Theta$ is linearizable by Poincaré Linearization Theorem. Thus given any point $q \in K$ we can choose an open set $U \ni q$ and local coordinates $(x, y, z) \in U$ centered at $q$ such that $\left.\mathcal{F}\right|_{U}$ is given by the closed meromorphic 1-form $\Omega_{U}=\frac{\lambda d x}{x}-\frac{d y}{y}$ and $K \cap U=\{x=y=0\}$. Suppose now that $\tilde{q} \in K$ is another point, $(\tilde{x}, \tilde{y}, \tilde{z}) \in \widetilde{U}, \Omega_{\tilde{U}}=\frac{\lambda d \tilde{x}}{\tilde{x}}-\frac{d \tilde{y}}{\tilde{y}}$ are chosen in the same way and $U \cap \widetilde{U} \neq \phi$. Then in $U \cap \widetilde{U}$ we have $\Omega_{\widetilde{U}}=f . \Omega_{U}$ for some meromorphic function $f$. Since $\Omega_{U}$ and $\Omega_{\tilde{U}}$ have order one polar divisors coinciding in $U \cap \widetilde{U}$ it follows that $f$ is holomorphic in $U \cap \widetilde{U}$ and since $0=d \Omega_{U}=d \Omega_{U}$ it follows that $f$ is a holomorphic first integral for $\left.\mathcal{F}\right|_{U \cap \tilde{U}}$. But since $\Omega_{U}$ and $\Omega_{\tilde{U}}$ do not depend on $z$ and $\tilde{z}$ it follows that $f$ does not depend on the variable $z$. Now, since $\lambda \notin \mathbb{Q}$, the the transversal type of $K$ does not admit a holomorphic first integral. It follows that $f$ is locally constant in $U \cap \widetilde{U}$. Finally since $\Omega_{\widetilde{U}}$ and $\Omega_{U}$ have residue equal to 1 along $\{x=0\} \cap U \cap \widetilde{U}=\{\tilde{x}=0\} \cap U \cap \widetilde{U}$ it follows that $f \equiv 1$ and therefore $\Omega_{U} \equiv \Omega_{\widetilde{U}}$ in $U \cap \widetilde{U}$.

Now we assume that $\lambda \in \mathbb{Q}$ and $\Theta$ is not linearizable (excluding this way case (1)). In this case it follows by Poincaré-Dulac Theorem ([5]), that $\lambda$ or $\frac{1}{\lambda} \in \mathbb{N}$ and we can write $\Theta=x d y-\left(n y+x^{n}\right) d x=x d y-\left(n y+x^{n}\right) d x=$ $x^{n+1}\left(d\left(\frac{y}{x^{n}}\right)-\frac{d x}{x}\right)$. Thus we define locally $\Omega=d\left(\frac{y}{x^{n}}\right)-\frac{d x}{x}$ and proceed as above. The glueing of these local models follows from the fact that $\Theta$ as above does not admit a local nonconstant meromorphic first integrals as it is easily verified using Laurent series.

If $\Theta$ is linearizable with $\lambda / \mu \in \mathbb{Q}_{+}$then we are in the situation of case (1) already considered.

- $\Theta$ is in the Siegel domain: $\lambda \in \mathbb{R}_{-}$. If $\lambda \notin \mathbb{Q}_{-}$, then (since $\Theta$ is analytically normalizable by hypothesis) the transverse type is linearizable and admits no meromorphic first integral. Using arguments similar to the ones used above we conclude that we may define closed 1 -forms with simple poles defining $\mathcal{F}$ in open sets $U$ that cover $K$ and such that $\Omega_{U} \equiv \Omega_{\widetilde{U}}$ in $U \cap \widetilde{U}$. 
Assume now that $\Theta$ admits a holomorphic first integral. We use the same notation as above. By Mattei-Moussu Theorem $\Theta$ is linearizable [16] and we have $\lambda / \mu=-n / m \in \mathbb{Q}_{-}, \quad n, m \in \mathbb{N},(n, m)=1$. Therefore we have $f(x, y)=\varphi\left(x^{n} y^{m}\right)$ for some holomorphic function $\varphi$. On the other hand, since the leaves of the transversal type are of the form $x^{n} y^{m}=c, c \in \mathbb{C}$, it follows that there are only two of these leaves which define separatrices namely $(x=0)$ and $(y=0)$. Therefore we can assume that $\widetilde{x}=u x$ and $\widetilde{y}=v y$ for some $u, v \in \mathcal{O}^{*}(U \cap \widetilde{U})$. It follows from $\Omega_{\widetilde{U}}=f \Omega_{U}$ that $\frac{n}{m} \frac{d x}{x}+$ $\frac{d y}{y}+\frac{n}{m} \frac{d u}{u}+\frac{d v}{v}=(f-1)\left(\frac{n}{m} \frac{d x}{x}+\frac{d y}{y}\right)=\left(\varphi\left(x^{n} y^{m}\right)-1\right)\left(\frac{n}{m} \frac{d x}{x}+\frac{d y}{y}\right)$. So that $\psi=u^{n} v^{m}$ is also a holomorphic first integral for $\left.\mathcal{F}\right|_{U \cap \mho}$. But this is a nonvanishing function so that necessarily we must have it locally constant and therefore $(f-1)\left(\frac{n}{m} \frac{d x}{x}+\frac{d y}{y}\right) \equiv 0$. Thus $f \equiv 1$. Again we have $\Omega_{U} \equiv \Omega_{\widetilde{U}}$ in $U \cap \widetilde{U}$.

Finally we assume that $\lambda / \mu \in \mathbb{Q}_{-}$but $\Theta$ is not linearizable. In this case since it is analytically normalizable, it follows that it is analytically conjugated to the Martinet-Ramis normal form [14] defined by $\omega_{n / m, k, \lambda}=$ $n\left[1+(\lambda-n l) u^{k}\right](n y d x+m x d y)+n \mu^{k}(l y d x+t x d y),, u=x^{n} y^{m}, \lambda \in \mathbb{C}, k \in$ $\mathbb{N},<n, m>=1$; where $l, t \in \mathbb{N}$ are such that $m l-n t=1$. Again we may define closed meromorphic 1-forms $\Omega_{U}$ in open sets that cover $K$. These 1 -forms $\Omega_{U}$ have nonsimple poles and admit no meromorphic first integral $[\mathbf{1 4}],[21]$ as it can be checked via Laurent Series, or by the use of [16]. Since the transverse type admits no meromorphic first integral these 1-forms $\Omega_{U}$ may be chosen in such a way that $\Omega_{U}=c t e . \Omega_{\widetilde{U}}$ in each $U \cap \widetilde{U} \neq \emptyset$. Arguing with residues along the $\operatorname{set} \operatorname{sep}(\mathcal{F}, K)$ we conclude that $\Omega_{U}=\Omega_{\tilde{U}}$ in $U \cap \widetilde{U} \neq \emptyset$ which proves this case. This ends the proof of (2).

Proof of (3). Given any point $q \in K$ we can choose an open set $U \ni q$ and local coordinates $(x, y, z) \in U$, centered at $q$, such that $\left.\mathcal{F}\right|_{U}$ is given by $\omega(x, y)=g\left(x\left(1+\lambda y^{n}\right) d y-y^{n+1} d x\right)$ for some meromorphic function $g$. We define $\Omega_{U}:=-\frac{d x}{x}+\lambda \frac{d y}{y}+\frac{d y}{y^{n+1}}$.

Claim 2.4. If $(\widetilde{x}, \widetilde{y}, \widetilde{z}) \in \widetilde{U}$ is another such system of coordinates with $U \cap$ $\widetilde{U} \neq \phi$, then $\Omega_{\widetilde{U}} \equiv \Omega_{U}$ in $\widetilde{U} \cap U$.

Proof of the claim. In $\widetilde{U} \cap U$ we have $g x y^{n+1}\left(\frac{d y}{y^{n+1}}+\lambda \frac{d y}{y}-\frac{d x}{x}\right)=g x y^{n+1} d(-$ $\left.\frac{1}{n y^{n}}+\lambda \log y-\log x\right)=\omega=\tilde{g} \widetilde{x} \widetilde{y}^{n+1}\left(\frac{d \widetilde{y}}{\widetilde{y}^{n+1}}+\lambda \frac{d \widetilde{y}}{\widetilde{y}}-\frac{d \tilde{x}}{\widetilde{x}}\right)=\widetilde{g} \widetilde{x} \widetilde{y}^{n+1} d\left(-\frac{1}{n \widetilde{y}^{n}}-\right.$ $\lambda \log \widetilde{y}+\log \widetilde{x})$.

Therefore $d\left(\frac{\tilde{g} \widetilde{x} \widetilde{y}^{n+1}}{g x y^{n+1}}\right) \wedge d\left(-\frac{1}{n y^{n}}+\lambda \log y-\log \widetilde{x}\right)=0$. Thus $\frac{g \widetilde{x} \widetilde{y}^{n+1}}{g x y^{n+1}}$ is a meromorphic first integral for the foliation $d\left(-\frac{1}{n \widetilde{y}^{n}}+\lambda \log \widetilde{y}-\log \widetilde{x}\right)=0$. 
However this last does not admit such a first integral (see [14] for instance) so that $\frac{\tilde{g} \tilde{x} \tilde{y}^{n+1}}{g x y^{n+1}}$ is locally constant in $U \cap \widetilde{U}$. This implies that $-\frac{d x}{x}+\lambda \frac{d y}{y}+$ $\frac{d y}{y^{n+1}}=a\left(-\frac{d \tilde{x}}{\tilde{x}}+\lambda \frac{d \tilde{y}}{\tilde{y}}+\frac{d \tilde{y}}{\widetilde{y}^{n+1}}\right)$ for some $a \in \mathbb{C}^{*}$. Comparing the residues along $(x=0)$ an $\mathrm{d}(\widetilde{x}=0)$ we conclude that $a=1$ proving the claim.

Now we state a first consequence of our approach (see [5]):

Theorem 2.5. Let $\mathcal{F}$ be a foliation on $\mathbb{C} P(k), k \geq 3$. Suppose there is a compact component $K \subset K(\mathcal{F})$ which is a transverse complete intersection. Then $\mathcal{F}$ has a rational first integral.

Proof. Let $\Theta$ be the transversal type of $K$. Using Theorem 1.1 (iv), we can assume that $\Theta=n x d y-m y d x, n, m \in \mathbb{N}$. According to Proposition 2.2 this implies that $\mathcal{F}$ is transversely projective in $V^{k}$ for some open neighborhood $V^{k}$ of $K$ in $\mathbb{C} P(k)$. Using [20],[21] and Proposition 2.2 we prove that $\mathcal{F}$ is transversely projective in $\mathbb{C} P(k)$. The proposition follows now from the fact that $\mathbb{C} P(k)$ is simply-connected (see [20],[21]).

This theorem is originally proved in [5] in a different way.

\section{Proof of the main results.}

Let $\mathcal{F}$ be as in Theorem B. Let $S=S_{1} \cup \cdots \cup S_{r}$ be the decomposition of $S$ in irreducible components. We recall that since $K(\mathcal{F}) \cap S$ is dense and open in $S$ and both $S, K(\mathcal{F})$ are analytic, it follows that $\operatorname{cod}_{S}\left(S_{j}-K(\mathcal{F})\right) \geq 1$, so that $S_{j} \cap K(\mathcal{F})$ is connected. We will denote therefore by $\Theta_{j}$ the transversal type of $\mathcal{F}$ at $S_{j} \cap K(\mathcal{F})$.

Lemma 3.1. Given any component $K \subset K(\mathcal{F})$ with transverse type $\Theta_{K}$, and a meromorphic integrable 1-form $\omega$ that defines $\mathcal{F}$, we have according to the possibilities of Proposition 2.2:

i) $\Theta_{K}$ is linearizable dicritical (i.e., of type (1): given any meromorphic 1form $\eta$ such that $d \omega=\eta \wedge \omega$, there exists a 1-form $\xi_{K}$ on a neighborhood $V_{K}$ of $K$ such that $\left(\omega, \eta, \xi_{K}\right)$ is a projective triple and defines a transverse projective structure for $\left.\mathcal{F}\right|_{V_{K}}$.

(ii) $\Theta_{K}$ is of type (2) or (3): there exists a neighborhood $V_{j}$ of $S_{j}:=$ $\bar{K} \cap S$ where it is defined a meromorphic 1-form $\eta_{j}$ such that $\left(\omega, \eta_{j}\right)$ is an affine pair. The pair $\left(\omega, \eta_{j}\right)$ defines an affine transverse structure for $\left.\mathcal{F}\right|_{V_{j} \backslash \operatorname{sep}(\mathcal{F}, K)}$. 
Proof. By the hypothesis if $q \in S \backslash K(\mathcal{F})$ then $\mathcal{F}$ is given by a closed meromorphic 1-form $\Omega_{q}$ in a neighborhood of $q$.

Proof of (ii). Fix a point $q^{\prime} \in K(\mathcal{F})$, close to $q$. This point $q^{\prime}$ belongs to a certain Kupka component $K$. Using Proposition 2.2 we obtain a closed meromorphic 1-form $\Omega_{K}$ which defines $\mathcal{F}$ in a neighborhood $\mathcal{U}_{K}$ of $K$. We may write $\omega=h_{K} \cdot \Omega_{K}$ and obtain this way a meromorphic function $h_{K}$ which is an integrating factor for $\omega$ in $\mathcal{U}_{K}$, that is, $\frac{1}{h_{K}} \cdot \omega$ is closed. We define the 1 -form $\eta_{K}$ in neighborhood $\mathcal{U}_{K}$ as $\eta_{K}=\frac{d h_{K}}{h_{K}}$. This is a closed meromorphic 1-form satisfying $d \omega=\eta_{K} \wedge \omega$. Moreover we have $\left(\eta_{K}\right)_{\infty}=$ $\operatorname{sep}(\mathcal{F}, K)$ and it has order one. We define $\eta_{q}=\frac{d g_{q}}{g_{q}}$ where $\omega=g_{q} \cdot \Omega_{q}$ in a neighborhood of $q$. The function $f=h_{K} / h_{q}$ is therefore a meromorphic first integral for the transverse type along $K$ : indeed, this is a quotient of integrating factors so, as it is easy to verify, we have $d f \wedge \omega=0$. Since the transverse type of $K$ does not admit a nonconstant holomorphic first integral it follows that $f$ is constant and then $h_{q}=$ cte. $h_{K}$ so that $\eta_{K}=\eta_{q}$ in a neighborhood of $q$.

Proof of (i). According to Proposition 2.2 (i), there exists a projective transverse structure for $\mathcal{F}$ in some neighborhood $\mathcal{U}_{K}$ of $K$. Thus it follows from [20],[21] that, given any rational 1-form $\eta$ satisfying $d \omega=\eta \wedge \omega$, we can find on $\mathcal{U}_{K}$ a meromorphic 1-form $\xi_{K}$ which satisfies $d \eta=\omega \wedge \xi_{K}$ and $d \xi_{K}=\xi_{K} \wedge \eta$. This triple $\left(\omega, \eta, \xi_{K}\right)$ defines the transverse projective structure for $\left.\mathcal{F}\right|_{\mathcal{U}_{(K)}}$.

According to Lemma 3.1 above, given any component $S_{j}$ of $S$ whose transversal type $\Theta_{j}$ is not of the (dicritical) form $n x d y-m y d x=0$ (that is, not of type (1) of Proposition 2.2), we can define a 1-form $\eta_{j}$ in a neighborhood $V_{j}$ of $S_{j}$ such that $\left(\omega, \eta_{j}\right)$ is an affine pair. Let $K_{i}$ and $K_{j}$ be two nondicritical Kupka components with $V_{i} \cap V_{j} \neq \phi$.

Lemma 3.2. For $\eta_{i}, \eta_{j}$ as above we have $\eta_{i} \equiv \eta_{j}$ in a neighborhood of $V_{i} \cap V_{j}$.

Proof. Indeed, we may assume that $\omega$ is not closed (otherwise $\mathcal{F}$ is already given by a closed meromorphic 1-form) and fix $q \in V_{i} \cap V_{j}$. As we have seen there exists a closed meromorphic 1-form $\eta_{q}=\frac{d h_{q}}{h_{q}}$ defined in a neighborhood of $q$ and satisfying also $d \omega=\eta_{q} \wedge \omega$. We have seen in the proof of Lemma 3.1 that, as a consequence of the fact that $K_{j}$ does not admit a meromorphic first integral, we have $\eta_{j}=\eta_{q}$ in a neighborhood of $q$. The same holds for 
$\eta_{i}$ so that we have $\eta_{i} \equiv \eta_{j}$ around $q$.

Proof of Theorem B. According to Lemma 2.1, conditions (b) and (c) imply that the the transverse type of any Kupka component is analytically normalizable so that we may apply Proposition 2.2 and Lemmas 3.1 and 3.2 in order to construct the affine pair $(\omega, \eta)$ in a neighborhood $V_{1} \cup \ldots \cup V_{r}$ of $S$ as in Theorem B. The last part is a consequence of the extension result Theorem 1.2.

Proof of Theorem C. Theorem C follows from Proposition 2.2 and Lemma 3.1 (i).

Proof of Theorem A. According to Theorem B we may construct $\eta$ in a neighborhood of $S$ in $\mathbb{C} P(k)$ such that $(\omega, \eta)$ is an affine pair. Using the extension result Theorem 1.2 we extend $\eta$ to a rational 1 -form in $\mathbb{C} P(k)$. Now, we know that $\eta$ is a closed rational 1-form that has simple poles and integer residues (for it is locally of the form $\eta=\frac{d h}{h}$ with $h$ meromorphic). Using the Integration Lemma [20],[5] (see Example 2) we can write $\eta=\frac{d h}{h}$ for some rational function $h$ on $\mathbb{C} P(k)$. The relation $d \omega=\eta \wedge \omega$ is equivalent to $d\left(\frac{1}{h} \omega\right)=0$.

Remark 3. According to a theorem of Sernesi ([22]) and to some stability theorems for regular singularities ([1]), we have that a small analytic perturbation of a foliation $\mathcal{F}$ having a quasiprojective complete intersection Kupka component (see Theorem A, condition (a)), and with regular singularities along this component, still exhibits a Kupka component with these same properties. However we recall that the normalization property of the transverse type may disappear.

We give now an application of our main result (Theorem A) to foliations on $\mathbb{C} P(2)$.

Theorem 3.3. Let $\mathcal{F}$ be a foliation having nondegenerate singularities on $\mathbb{C P}(2)$ and assume that the singular set $\operatorname{sing}(\mathcal{F})$ is a complete intersection of two algebraic curves $C_{1}$ and $C_{2}$. Assume also that the singularities of $\mathcal{F}$ are analytically normalizable, and nonresonant. Then $\mathcal{F}$ is given by a closed rational 1-form $\Omega$ on $\mathbb{C} P(2)$.

Proof. Given the foliation $\mathcal{F}$ we consider a linear embedding $\mathbb{C} P(2) \rightarrow$ $\mathbb{C} P\left(\right.$ ?) and for a point $p_{o} \in \mathbb{C} P(3) \backslash \mathbb{C} P(2)$ we construct the "cone" $\widetilde{\mathcal{F}}$ induced by $\mathcal{F}$ on $\mathbb{C} P(3)$ in the usual way having $p_{o}$ as vertex. It is clear that 
$\widetilde{\mathcal{F}}$ satisfies the hypothesis of Theorem A (the point $p_{o}$ will be a simple singularity in the sense of [1]). Therefore we can obtain a closed meromorphic 1 -form $\widetilde{\Omega}$ which defines $\widetilde{\mathcal{F}}$ on $\mathbb{C} P(3)$. The restricted 1-form $\Omega=\left.\widetilde{\Omega}\right|_{P(2)}$ is closed and rational, and defines $\mathcal{F}$.

\section{References.}

[1] C. Camacho and A. Lins Neto, The topology of integrable differential forms near a singularity; Publ. Math. I.H.E.S., 55 (19Ca-Ce-Sc2), 5-35.

[2] C. Camacho and P. Sad, Invariant varieties through singularities of holomorphic vector fields; Ann. of Math. 115 (1982), 579-595.

[3] F. Cano, D. Cerveau, B. Azevedo Scárdua, Holomorphic foliations desingularized with punctual blow-ups. Pre-print, I.R.M.A.R.- Rennes I, November 1997.

[4] D. Cerveau, A. Lins Neto, Irreducible components of the space of holomorphic foliations of degree two in $\mathbb{C} P(n), n \geq 3$; Ann. of Math. (1996) pg. $577-612$.

[5] D. Cerveau, A. Lins Neto, Codimension-one foliations in $\mathbb{C} P(n) n \geq 3$ with Kupka components; Astérisque 222 (1994), 93-133.

[6] D. Cerveau, J.-F. Mattei, Formes intégrables holomorphes singulières. Astérisque, vol.97(1982).

[7] X. Gomes-Mont, A. Lins Neto, Structural stability of foliations with a meromorphic first integral; Topology 30 (1991), 315-334.

[8] H. Grauert, R. Remmert : Theory of Stein Spaces; Springer-Verlag.

[9] R.C. Gunning: Introduction to Holomorphic Functions of Several Variables; Vol. I; Wadsworth \& Brooks/Cole.

[10] R. Gunning \& H. Rossi: Analytic functions of several complex variables; Prentice Hall, Englewood Cliffs, NJ, 1965.

[11] I. Kupka, The singularities of integrable structurally stable Pfaffian forms, Proc. Nat. Acad. Sci. U.S.A., 52 (1964), 1431-1432.

[12] A. Lins Neto, Finite determinacy of germs of integrable 1-forms in dimension 3 ( A special case). Geometric Dynamics, Springer LNM no. 1007, pp.480-497, (19Ca-Ce-Sc3). 
[13] A. Medeiros, Structural stability of integrable differential forms, Geometry and Topology (M. do Carmo, J. Palis eds.), LNM, 1977, 395-428. no della Unione Matematica Italiana, vol. 12 (1975), 138-146.

[14] J.Martinet et J-P.Ramis, Classification analytique des équations différentielles non linéares résonnantes du premier ordre. Ann. Sci. Éc. Norm. Sup. , t 16, 1983, 571-621.

[15] J. Martinet et J-P.Ramis, Problème de modules pour des équations différentielles non lineares du premier ordre, Inst. Hautes Études Sci. Publ. Math. 55 (1982), 63-124.

[16] J.F. Mattei and R. Moussu, Holonomie et intégrales premières; Ann. Ec. Norm. Sup. 13 (1980), 469-523.

[17] J. Omegar, Persistência de folheações definidas por formas logaritmicas. Thesis, IMPA-Rio de Janeiro, 1990.

[18] J. Omegar, Irreducible components of the space of holomorphic foliations. Math. Annalen, no. 299, pp.751-767 (1994).

[19] G. Reeb, Sur certains propriétés topologiques des variétés feuilletées. Actual. Sci. Ind. \# 1183, Hermann Paris, 1952.

[20] B. Azevedo Scárdua, Transversely affine and transversely projective holomorphic foliations. Ann. Sc. E.N.S., t.30 (1997), 169-204.

[21] B. Azevedo Scárdua, Integration of complex differential equations. Journal of Dynamical and Control Systems $\geq 1998$.

[22] E. Sernesi, Small deformations of global complete intersections, Bollettino della Unione Matematica Italiana, vol. 12 (1975), 138-146.

[23] M. Spivak : A Comprehensive Introduction to Differential Geometry; Vol. 1, second edition, Publish or Perish, Berkeley, 1979.

[24] Y.Siu, Techniques of Extension of Analytic Objects. Marcel Dekker, New York, 1974. 
RECEIVEd JuLY 2, 1997.

IMPA

Est. D. CAstorina 110

22460-320 RIO DE JANEIRO

BRAZIL 